\title{
Home energy monitors: impact over the medium-term
}

\author{
S.S. van Dam (corresponding author) \\ Delft University of Technology \\ Faculty of Architecture \\ Julianalaan 134
}

2628 BL Delft, the Netherlands

s.s.vandam@tudelft.nl

\section{C.A. Bakker}

Delft University of Technology

Faculty of Industrial Design Engineering, dept Design for Sustainability

Landbergstraat 15

2628 CE Delft, the Netherlands

c.a.bakker@tudelft.nl

J.D.M. van $\mathrm{Hal}$

Delft University of Technology / Nyenrode Business Universiteit Julianalaan 134 / Straatweg 25,

2628 BL Delft / 3621 BG Breukelen

The Netherlands

j.d.m.vanhal@tudelft.nl

This is an electronic version of an article published in van Dam, S. S., Bakker, C. A. and van Hal, J. D. M.(2010) 'Home energy monitors: impact over the medium-term', Building Research \& Information, 38: 5, 458 - 469. Building Research \& Information is available online at: http://www.tandfonline.com/openurl?genre=article\&issn=0961$\underline{3218 \& \text { volume }=38 \& \text { issue }=5 \& \text { spage }=458}$ 


\begin{abstract}
Home Energy Management Systems (Hems), e.g. energy monitors, are intermediary products that can visualize, manage, and/or monitor the energy use of other products or whole households. Hems increasingly receive attention for their role in energy conservation in households. A literature review and a case study examine the mid-term effectiveness (> 4 months) of Hems. The case study present the results of a 15-month pilot with a domestic energy monitor in the Netherlands. It explores the extent to which participants manage to sustain their initial electricity savings over time, with a special focus on the development of habitual energy saving behaviour. The results show that the initial savings in electricity consumption of $7.8 \%$ after 4 months could not be sustained in the medium to long term. A second finding is that certain groups of people seem more receptive to energy saving interventions than others. These participants quickly develop new habits and exhibit larger savings than other participants. Obviously, a 'one-size-fits-all' approach for home energy monitors cannot be justified. For Hems to be effective, a deeper understanding is needed embracing social science, contextual factors, usability and interaction design research.
\end{abstract}

\title{
Keywords
}

Energy demand, energy management, housing, feedback, energy monitor, Home Energy Management System, habits, occupant behaviour, human agency, smart metering 


\section{Introduction}

Residential electricity consumption has been growing in all regions of the world at an average of $3.4 \%$ per year since 1990 . Although some of this growth is a result of more people with access to electricity, the majority is caused by the increased consumption of electricity by individual households (International Energy Agency (IEA), 2009). Western households commonly own a growing number of appliances that currently range from 28 to 67 (Milieu Centraal, 2005, IEA, 2009). Also, the number of technical installations for space heating, cooling and ventilation is increasing. Technological solutions, such as switching to the most energy efficient technologies available, could save up to $40 \%$ in residential electricity consumption(IEA, 2009). However, the focus on technology alone appears to have its limitations. With regards to energy, for instance, occupant behaviour was found to be the major contributor to the variance in domestic energy consumption (Brohus et al., 2009, Crosbie and Baker, 2010) As Crosbie and Baker (2010) say: "It does not matter how much energy hypothetically could be saved by efficient technologies if no one wants to live in the properties, install or use efficient lighting and heating." In the endeavour to tackle this behavioural dimension, smart metering and Home Energy Management Systems (Hems) are being given increasing attention, both in academia and in commercial enterprises. Hems are much advertised as 'high potentials' for domestic energy savings, with some (commercial) energy monitors claiming 10 to $20 \%$ savings. For the authors, this was the starting point for a critical examination of these seemingly optimistic claims.

Hems are defined as intermediary devices that can visualize, monitor and/or manage domestic gas and/or electricity consumption. Their main purpose is to give users direct and accessible insight into their energy consumption. This makes them different from smart meters which are predominantly intended to benefit the gas or electricity supplier and generally need a Hems to give users the intended insight. Hems come in many shapes and sizes and vary in six significant areas; the first area is the 
type of energy they are intended for; gas and/or electricity. Second, they differ at the level at which feedback is given. Some give feedback on overall household (utility) consumption, some break it down to separate appliances (disaggregated), and yet others limit feedback to just one appliance. Third, they vary according to the type of feedback they provide, such as factual (e.g.. showing real-time consumption data, figure 1), social (e.g. using

\section{5 i

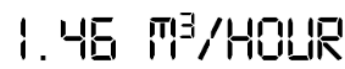

\section{Figure 1 factual Feedback}

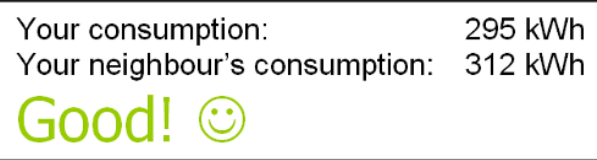

Figure 2 Social (comparative) Feedback

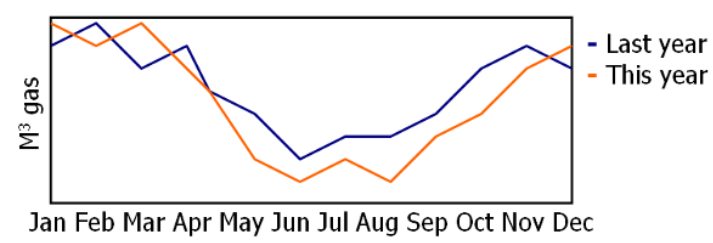

\section{Figure 3 historical comparative feedback}

smiling/frowning faces, figure 2), or comparative (e.g. current versus historical consumption data, figure 3). A fourth characteristic is whether they only monitor or also manage energy consumption. The difference is that monitors only give feedback, leaving it to the user to decide whether or not to act on the feedback, while managers help users control if and when their appliances consume energy. Fifthly, the type of interaction and physical location of Hems varies from local appliance-specific solutions, such as standby-killers, to central inhome touch screens, to online web-applications. And finally, their intended purpose can be energy saving or peak shaving (peak shifting of the grid load).

The type of Hems that has been commercially available since the seventies is the energy- or 
electricity monitor, which solely gives inhabitants feedback on electricity consumption at utility (household) level. This energy monitor has been dominating the market for years, and it is also the type of Hems that has featured most in academic research. Recently, other kinds of Hems have entered the marketplace and future studies will hopefully broaden their perspective to include these.

\section{Research objectives and method}

The general objective of this paper is to achieve a better understanding of the effectiveness of Hems. A literature review will focus on Hems in general whereas the second part of this article, the case study, will focus on energy monitors, considering the medium-term ( $>$ four months). We define effectiveness as the extent to which users can maintain significant energy savings over these prolonged periods.

Most of the studies into Hems have asked the same basic question: how effective are they in helping people save energy? Although this question has been answered many times and often with a positive result (for instance: Ueno et al., 2006b, Midden and Ham, 2009), the majority of these studies have only assessed the use of energy monitors over relatively short periods (four months or less). The few studies that have taken a long(er) view show indecisive results (van Houwelingen and van Raaij, 1989). This uncertainty is the focus of the paper. What are the medium- to long-term results of Hems on energy savings? What happens to the energy savings after the initial four months of Hems usage? What is the influence of the design quality and usability of Hems? Is there a relationship between the amount of Hems usage and achieved energy savings, and what role does the development of habitual behaviour play? This paper explores these questions through a literature review and a case study.

\section{Literature review}

\section{Background relations}


Background relations with technology are described from a philosophical point of view by Inde (1990) who identifies products with an 'absent presence' in households. They operate in the 'background' either physically or in the back of our minds. Even more so, an increasing number of these background appliances, especially technical installations, function autonomously. They self-regulate their energy use which is consequently imperceptible to users. While some products might drift into the background over time, most background products are intentionally designed to operate this way: There is deliberately little to no interaction with the end user. Borgmann (1995) describes this as disburdening but also disengaging technology. As a negative side effect, these background products significantly contribute to the energy consumption of households and the invisibility of energy flows in homes. More than half of $\mathrm{CO}_{2}$ emissions of households are caused by background appliances and 'imperceptible' energy consumption like phantom loads (derived from Milieu Centraal, 2008).

While the disburdening effect also brings important beneficial aspects to users, it tends to undermine the direct cause and effect relationship between users, their behaviour and energy consumption. This is where Hems come in: In their mediating role, they provide people with a (visual) representation of the energy consumption, and help them mentally interpret the actual energy (or monetary) figures and perceive the energy consumption of other products. Ihde (1990) calls this relationship between users and products a hermeneutic relationship. An alterity relationship between users and Hems takes place when the Hems itself is the continual focal point of attention. However, some instances have been recorded where Hems slowly drift into the background of people's attention, thus undermining their core purpose of giving recurrent feedback and ultimately becoming obsolete. When a Hems drifts into the background, a shift from alterity relation to background relation takes place. Several case studies have shown this change from alterity to background. The most detailed studies were done by Ueno et al. (2006a, 2006b). These reported drastic reductions in the usage of a display giving disaggregated feedback during the first two to four weeks, after 
which stabilization took place. In the three-month pilot of the 'PowerPlayer' (Firet L.T., 2009) participants indicated that at a certain point in time they understood their gas and electricity usage patterns, and used the 'PowerPlayer' less. Respondents did add that they would use the monitor again when buying new appliances. In a study on the use of an electricity monitor (PowerCost) by Mountain (2006), one third of the participants indicated they would not use the monitor any more after the pilot. Lofstrom and Palm (2008) addressed the use of the Power-Aware Cord, which can visualize the electricity use of a single socket. They tested it for two months with six households and found that whereas in the beginning people were enlightened by the energy use of an appliance, after some time the Power-Aware Cord 'just' became a decorative element, likened by all participants to Christmas lights.

Although it is impossible to draw decisive conclusions based on these studies, the general trend seems to be that feedback devices slowly drift into the background. The exact cause of this finding has not been studied, although it is conceivable that people simply lose interest. This raises the question how to prevent Hems from drifting into the background. One possible answer lies in the increased interaction with Hems, e.g., when people develop habits around them. Another possibility is the improved design of Hems and their feedback, as several design strategies have been created to influence behaviour through products (Lockton et al., 2008). A third option would be to view a background relation as a potential strength by implementing Hems that not only monitor but also manage energy. Energy managers operate more in the background, programmed (by users) to switch off appliances that are not in use. They are therefore less dependent on behavioural change for energy savings than monitors. Whereas monitors only give feedback, energy managers additionally give participants the ability to control their energy consumption. Perceived control is claimed to have a positive influence on users (Geller, 1995 and Allen and. Ferrand, 1999), although raising awareness through feedback still remains essential. However, there has been no scientific research into energy managers to validate their effectiveness (especially in comparison to energy monitors). This is therefore a potential area for future research. As past studies do give some 
insight into the possible role for design and habits, these will be discussed in the next sections, starting with habits.

\section{Habits}

Within psychology, habits are mainly approached as a barrier which prevent the development of new behavioural patterns and act as a cause of fallback. Existing habits, such as leaving the lights on, are seen as major obstructions to the development of new, sustainable behaviours. Even after months of trying to adopt a new pattern, users can still fall back into the old pattern. Habits take time to develop. Therefore studies on feedback, intent on changing energy related behaviour, need to have a long term perspective. However, most of the studies on feedback were conducted over a period of less than four months and often without follow-up (for an extensive review see Abrahamse et al., 2005). In the rare cases that medium to long-term studies have been conducted, the outcomes were indecisive (van Houwelingen and van Raaij, 1989, Mountain, 2006) or the studies failed to record long term energy reduction results (Ueno et al., 2006a).

A different approach to habit development is studying the development of new habits around Hems so that users receive recurrent feedback. In this approach, habits are used as a strategy to prevent feedback devices from drifting into the background. While drifting into the background alludes to decreased use, to the point of abandonment, habitual use implies ingrained, regular use. Past research has not given specific attention to habitual use of Hems, but it is apparent in some studies that under a limited group of users, regular usage does take place at any rate. In Mountain (2006) 38.9\% of the participants in the case study indicated they looked at the PowerCost monitor at least once a day. Kidd and Williams (2008) describe how participants develop certain habits around the Efergy monitor. Matsukawa's (2004) study implies a relationship between amount of use of the energy monitor and energy saving. Though by no means a conclusive answer, it does warrant further research on the question if a relationship between (habitual) use of Hems and savings exists. 
While it can be left to chance whether users develop habits around Hems, it can also be part of Hems's design strategy. For their design to enable habits, they need to fit into people's daily lives and users should want to use them. Attention for design is not only beneficial to habit development; it is also important for its influence on effectiveness, which brings us to the next topic.

\section{Research on feedback and the design of Hems}

When discussing the influence of design on the effectiveness of Hems and prevention of nonuse, it is important not only to focus on the physical appearance. Associated elements such as type of feedback, the architecture, the interface, interaction between users and product, and the context in which it is used, need to be included. As feedback is the core functionality and dominant 'behaviour intervention mechanism' in most Hems, it poses a good starting point for the discussion. While feedback is approached by social scientists as strictly a behavioural intervention, it is inherently intertwined in the design of Hems. A designer would consider certain aspects of feedback, e.g. presentation style, a smiley or the colour usage, as a means of making the interface understandable for the user.

Several researchers have reviewed studies on feedback. In their review, Abrahamse et al. (2005) conclude that feedback tends to be more effective when combined with other strategies, and also with increased frequency (e.g. real-time feedback instead of feedback at fixed points in time). Darby (2006), in reviewing the effectiveness of feedback, comes to the same conclusion: immediate direct feedback could be extremely valuable, as long as it is provided through a user-friendly display. Likewise, Fischer (2008) concludes that the most successful feedback should be given frequently and over a long time, provide an appliancespecific breakdown, and be presented in a clear and appealing manner using interactive tools. Mccalley and Midden (2002), in much the same vein, conclude that product-integrated 
feedback, when coupled with the possibility to set an energy conservation goal, is a potentially highly successful means to save energy.

Some studies have found that people use feedback devices to track down a particular appliance at a specific point in time that is causing the rise in energy consumption (Kidd and Williams, 2008). One such study classified users accordingly as 'seekers, detectives, and judges' (Liikkanen, 2009). Knowing this, designing Hems to give accurate and real-time feedback becomes essential. Likewise, considerations should be given whether to design portable or fixed Hems, and if they should give disaggregated feedback. With energy monitors that only give feedback on overall household consumption, tracing back energy consumption to individual appliances can provoke a single-minded concentration on peaks by users (Kidd and Williams, 2008). The pitfall is that users consequently neglect continuous lower energy consuming appliances that use more in the long run. Also, attention should be paid to delays or infrequent data transmission, as this can lead to inexplicable peaks for users. Kidd and Williams' (2008) study noted furthermore that some participants switched types of energy being used, for instance heating water on the gas stove rather than an electric kettle. Feedback on both gas and electricity consumption is therefore desirable, in particular given the fact that gas is responsible for a larger proportion of $\mathrm{CO}_{2}$ emissions of households in the Netherlands. (EnergieNed, 2007) In other words, these examples show that the design of the feedback given by Hems should afford a certain type of use.

Feedback is one element in the design of Hems. This section discusses to what extent researchers have taken into account other aspects of the design, in particular the design of the human-Hems interaction. This human-product interaction concerns the 'use, understanding and experience' of products. This is broader than the straightforward use of a product as it includes the 'physical, cultural, technological, and societal contexts' in which they are used (DUT, 2009). 
The assumption is that using specific design strategies and design knowledge can help make Hems more effective, particularly in the long run. Although some researchers acknowledge the importance of a user-friendly display (see previous section), few studies actually report implementing knowledge from the design of human-computer interaction $(\mathrm{HCl})$ field. Commercially available monitors have a predominantly 'high-tech' design, and studies regularly implement these monitors. This can lead to usability problems, as was documented for one participant with the use of the Efergy monitor (Kidd and Williams, 2008): "I certainly haven't used it ... I certainly am not techno...".

One journal article on Hems by Wood and Newborough (2006), does detail a number of $\mathrm{HCl}$ aspects to consider when designing what they call 'energy consumption indicators'. In answering five questions concerning measurement units, categorisation and visualization of information, and the amount of possible interaction, Wood and Newborough emphasis the need for consideration of users and their use of Hems to achieve energy savings. Outside the field of Hems, in for example interaction design research, new strategies for influencing behaviour through design have also been developed in recent years. One such approach looks at 'persuasive techniques'. There are numerous similarities in the way people interact with each other and the way they interact with (the interface of) a product. People talk to products, show affection, or get angry with products, just as they do with other people. The manner in which products are designed can effect people's emotions and the manner in which they act. Knowing this, it is but a small step to reason that influence tactics could be integrated into products to persuade people into acting in a certain way. This has been coined 'captology' by Fogg (2003) who has also developed a behaviour change model (Fogg, 2009) to assist designers in creating persuasive products. Motivation, ability and trigger are the three principle factors herein. Merging persuasion theories and combining them with work from other (design) fields, Lockton $(2008,2009)$ is developing the Design with Intent (Dwl) method. Dwl is defined as 
"design that's intended to influence, or result in, certain user behaviour" through six 'lenses' or strategies, that can motivate, enable or constrain a certain behaviour. Persuasive technology and Dwl techniques are valuable for the design of the interface and architecture of Hems. It can help strengthen habits, increase usage, and heighten effectiveness. Implementing Fogg's line of thinking into Hems, the first step would be to create the right triggers at the right moment. Another step could be to increase the simplicity to heighten people's ability to save energy. Like Fogg Cialdini (1993), teaches that the manner in which something is presented to a user is very significant, but has given particular attention to normative social influence (the importance of what other people do) in recent years (Goldstein et al., 2008). Hems are well suited to compare the consumption of a household to that of other households and in doing so implement both descriptive and injunctive social norms through different visualization techniques. Knowing which strategy is the most effective and which is preferred by different types of user, is complicated however and needs to be studied in more detail.

\section{Conclusions from literature review}

This review poses directions for future research. It highlights several focal points that deserve more attention and are key to creating effective Hems and reducing energy consumption. Background appliances are an important factor in energy consumption, which makes accurate, traceable feedback valuable. Additionally the usability, with instances of decreased use and non-use of feedback devices needs to be addressed. Implementing insights from interaction design to develop user-friendly interfaces can help. But this needs to become a focal point in research and research needs to study various types of Hems, e.g. monitors as well as managers, to compare effectiveness. Finally, there is too little attention for medium to long-term research. This is needed to see whether energy saving behaviours become lasting habits and whether routine use can contribute to increased effectiveness. Not all can be studied within one research though, but the following case study will focus on one aspect; 
medium-term effectiveness, in relation to habitual use of energy monitors.

\section{Case study: 15-month pilot with a home energy monitor}

\section{Introduction}

In 2008/9, a 15-month pilot took place in the Netherlands. The pilot consisted of a four-month initial trial, instigated by several commercial parties to assess the effectiveness of a newly developed home energy monitor, and a follow-up study by TU Delft. The follow-up study took place 11 months after the initial trial ended, making the total trial period 15 months. The case study was anonymized, due to sensitivities surrounding the commercialization of this newly developed product.

The energy monitor consists of a sensor, a sending unit and a display. The sensor and sending unit are attached to the electricity meter. The sending unit sends a radio signal to the display unit. The display has three settings. In its standard setting it shows the power consumption in Watts in real-time (with a short delay of up to 10 seconds). It can also indicate daily consumption (over the past 24 hours), and can compare daily consumption with a personal savings target. The daily target has been corrected to the individual's fluctuations in consumption throughout the week. The monitor was designed to be simple in use, and users acknowledged this as only $5 \%$ gave the monitor a 'negative' or 'very negative' score on ease of use after installation. A website with energy-saving tips accompanies the monitor, as well as a voluntary email service ('learn and save') with more tips.

The objectives of this case study were firstly, to gain insight in the effectiveness of the energy monitor in the eleven months after the initial four month pilot. The effectiveness was defined as the extent to which having the monitor at home contributed to sustained electricity savings. The second objective was to explore whether a habit of daily checking the monitor had a positive influence on sustaining the electricity savings over the trial period. Based on results from previous studies (see literature review), we had certain expectations 
and assumptions about the outcomes of this case study. We expected the electricity savings to be considerable after the four-month initial trial, consistent with other short-term research. However, we expected that over a longer period some of the initial savings would be lost as a result of the monitor 'drifting into the background'. We also expected that people who developed a habit around the monitor (checking it at least once a day at fixed times) would better manage to sustain their electricity savings, as opposed to people who did not develop habitual behaviour.

The resulting hypotheses are therefore:

- At the end of the initial four-month trial, participants have an overall mean electricity saving.

- Participants' initial (four-month) electricity savings is not expected to be sustained over the entire 15-month period. However, participants who kept the monitor at home after the four-month initial trial are expected to better sustain their electricity savings than participants who returned the monitor.

- There is a positive relationship between having a daily habit of checking the monitor and sustaining the electricity savings over the 15 -month period.

\section{Method}

Participation in the case study was on a voluntary basis. The initial four-months trial started in the last week of June 2008 and ran until the first week of November 2008. A total of 304 participants received an energy monitor with instructions for its use and installation. Most of the participants also filled out the three online surveys that were sent out at the beginning, halfway and end of the trial. As a reward for their participation, at the end of the four-month period, people were given the option to either keep the energy monitor or return the monitor and receive a gift certificate of $€ 25$ instead. 


\begin{tabular}{|l|l|l|l|}
\hline Follow up study & Nr of participants & Kept monitor & Returned monitor \\
\hline Raw data & 189 & 93 & 96 \\
\hline All electricity data available & 54 & 26 (group A) & 28 (group B) \\
\hline
\end{tabular}

Table 1. Overview of number of participants in follow-up study (4-15 months).

In September 2009, 11 months after the initial trial, 264 participants who had filled out at least one of the surveys received an email asking them to participate in an online follow-up questionnaire. Of the 189 respondents, 93 had kept the monitor after the four-months trial, and 96 had returned it. The electricity meter readings of the 189 respondents were checked for completeness and inconsistencies. All meter readings were self-reported at five different occasions approximately 1 year before and during the case study, which unfortunately introduced quite a large number of reporting errors and missing data. Participants with incomplete data were eliminated, as well as participants with changes in family circumstances (e.g. moves, divorces, babies, children moving out). This left 54 participants for whom all meter readings were present and consistent. Of these, 26 had kept the monitor after the initial trial, and 28 had not. Within the group who had kept the monitor (group A) a distinction was made between those who had developed a daily habit after 15 months and those who had not (see table 1 and 2).

The electricity consumption during baseline, initial trial and follow-up was corrected for seasonal influences and extrapolated to yearly consumption.

\begin{tabular}{|l|l|}
\hline Group A & Nr of participants (total = 26) \\
\hline A, with daily habit $(\mathrm{AH})$ & 14 \\
\hline A, without daily habit $(\mathrm{ANoH})$ & 12 \\
\hline
\end{tabular}

Table 2. Subdivision of group A.

\section{Findings}


From the questionnaires and the meter data a number of results were obtained. First the main results from the questionnaires are discussed after which an analysis of the meter data is elaborated on.

\section{Demographics}

The demographics showed no exceptional data: The baseline consumption of $3614 \mathrm{kWh}$ was the same as the Dutch average (which increases 1.1\% per five years) (EnergieNed, 2006), while the household size of 2.4 was slightly above the Dutch average of 2.3. The large majority $(n=43)$ was home owner. Household size, income, and tenure were spread evenly between groups $A$ and $B$.

\section{Habits}

The responses to the questionnaires $(\mathrm{N}=189)$ were analyzed to understand the process of habit formation with the monitor. Of the 93 respondents who kept the monitor (table 3), 80 indicated they still had a functional monitor in their homes, which was also in use. Seventeen of the 80 respondents indicated they used the monitor less than during the initial four-month trial, but 53 respondents said they checked it daily at a fixed moment in time.

\begin{tabular}{|l|l|}
\hline Participants with functional energy monitor & 80 \\
\hline Use monitor less during follow-up & 17 \\
\hline Report habitual behaviour & 53 \\
\hline
\end{tabular}

Table 3. Majority of participants develop habitual behaviour.

If one looks specifically at the habits that were formed, then checking the monitor before going to bed was by far the most common routine. Second most popular was after getting up in the morning. Less common were before going out, or while using a specific appliance or space. It is interesting to note that the living room was by far the most common place to have 
the monitor, followed by the kitchen. None of the participants had chosen to place the monitor in the bedroom. Knowing that checking electricity consumption before going to bed is a common ritual can have design implications. Designers could consider fitting an 'energy check' into an existing bedtime ritual, such as turning down the thermostat, turning off the T.V. or lights, locking up the house, or setting the alarm clock. This could influence the monitor's design and functionalities as well as its favoured location.

\section{Savings}

To understand the effects of the interventions with the energy monitor, the electricity savings were studied. A first observation is that household electricity savings varied widely (figures 4 and 5). The household that saved the most (a family of three) saved $42.6 \%$ electricity during the pilot and $30.4 \%$ during the follow-up. By contrast, the worst performer (a family of two) used $33.6 \%$ more electricity during the initial trial and $40.6 \%$ more during the follow-up. The reasons for these extremes were quite straightforward. The 'high spender' (initial electricity consumption of $3985 \mathrm{kWh} /$ year) had just purchased an air conditioner, whereas the 'top saver' (initial electricity consumption of $2673 \mathrm{kWh} /$ year) followed a strict regime: writing down electricity meter data twice a day, recording how often washing machine and dishwasher were used, replacing all incandescent bulbs, limiting the use of the tumble dryer to bare necessity, decreasing the use of the dishwasher, and placing a timer on the pump of the garden pond. He made an extra note that he was 'very proud' of his achieved savings. By far the most commonly noted responses for expected savings with other participants were less use of appliances and unnecessary lighting and switching appliances off rather than in standby. CFLs and LED lights were also an easy target. Buying new energy saving appliances sometimes occurred, but was mentioned less often and mainly consisted of new washing machines, fridges and freezers. One participant purposefully noted not buying a flat screen TV. In a couple of instances a spill-over effect to gas consumption could also be noted as a participant turned their heater lower, adjusted the thermostat time schedule, or 
installed double glazing. Whether this was directly caused by the interventions can not be confirmed.

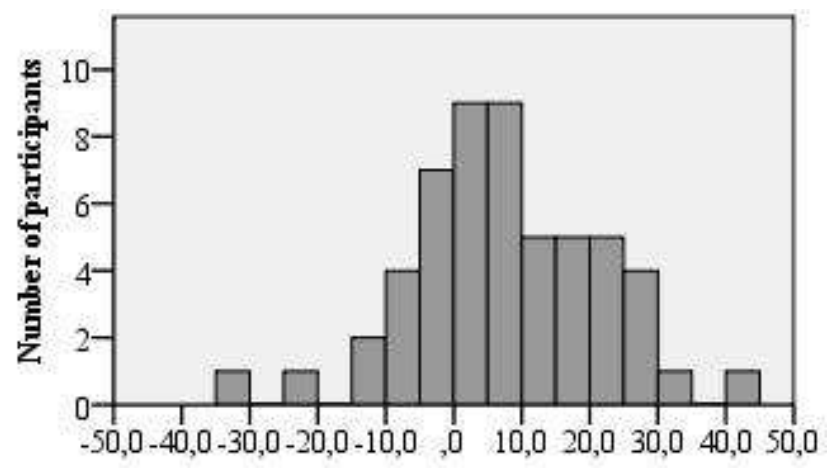

Figure 4. Histogram of achieved savings (\%) after 4 months (Mean=7.8 SD=13.8, N=54).

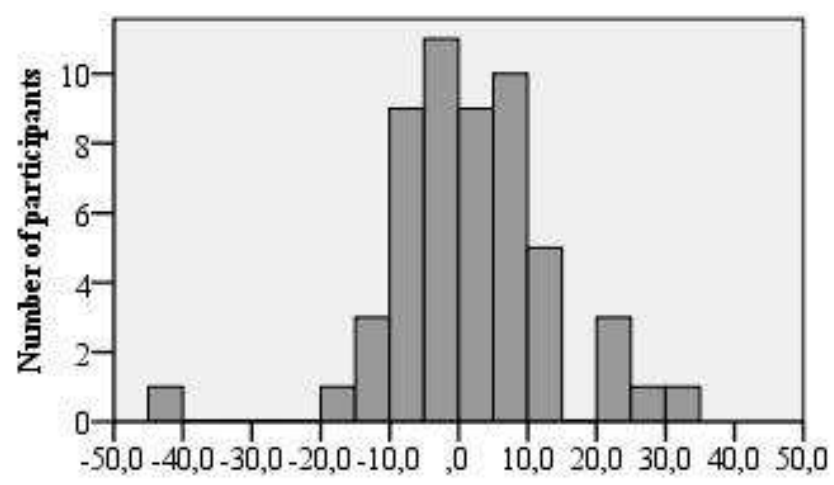

Figure 5. Histogram of achieved savings (\%) after 15 months (Mean=1.9 SD=11.8, $\mathrm{N}=54)$.

To further analyze the data, a mixed between-within subjects ANOVA was conducted to assess whether there was a difference in percentage of electricity saved, across two time periods (four-months trial, and eleven-months follow-up). The participants were split up in three groups:

- Group A consisted of participants who had kept the monitor after the initial trial, and was subdivided in two subgroups:

- Participants who had developed a habit (AH),

- Participants who had not developed a habit (ANoH). 
- Group B was the group that had returned the monitor after the initial four-month trial.

The electricity savings were corrected by using the baseline consumption as covariate. This was done to correct for large variances in individual electricity consumption of households. There was no significant interaction effect between the three conditions and the time (Wilks lambda $=.97 F(2,50)=.67 p=.52)$. This means that the savings of all 3 groups $(\mathrm{AH}, \mathrm{ANoH}$ and B) declined at the same rate during the course of the follow-up, in spite of the steeper decline that might seem apparent for $\mathrm{AH}$ from figure 6 . On the basis of the second hypothesis it was expected that the fallback would be less for participants who had kept the monitor, and in particular for those who had formed a habit.

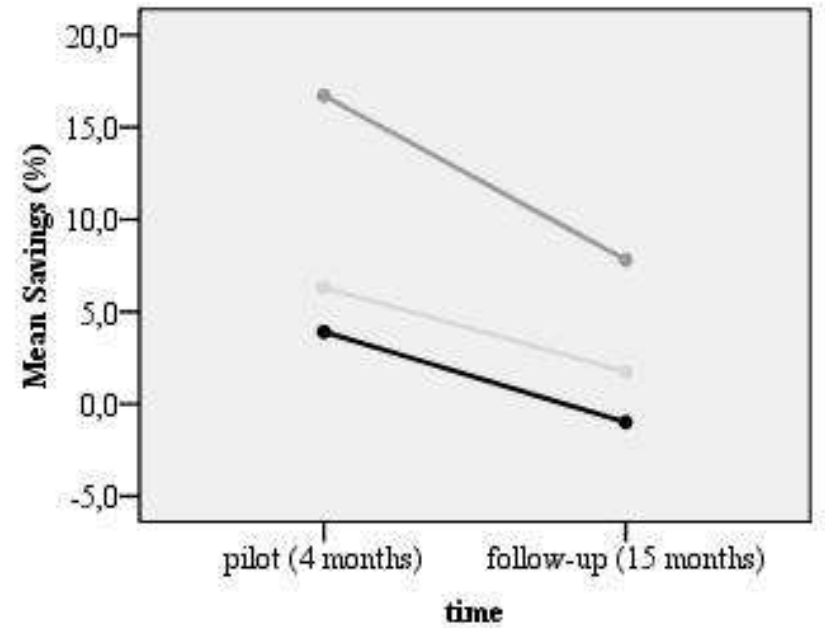

Legend

- AH: Kept energy monitor with daily habit

ANoH: Kept energy monitor without daily habit

-B: Retuned energy monitor after 4 months

Figure 6. Mean Savings (\%) after 4 and after 15 months. 


\begin{tabular}{|c|c|c|c|c|}
\hline \multirow[b]{2}{*}{ Condition } & \multirow[b]{2}{*}{ Time } & \multirow[b]{2}{*}{ Mean } & \multicolumn{2}{|c|}{ 95\% Confidence Interval } \\
\hline & & & Lower Bound & Upper Bound \\
\hline ANoH: kept monitor but no daily habit $(\mathrm{N}=12)$ & $\begin{array}{l}\text { Pilot } \\
\text { Follow-up }\end{array}$ & $\begin{array}{l}6.320^{\mathrm{a}} \\
1.740^{\mathrm{a}}\end{array}$ & $\begin{array}{l}-1.198 \\
-4.985\end{array}$ & $\begin{array}{l}13.838 \\
8.464\end{array}$ \\
\hline B: handed monitor in $(\mathrm{N}=28)$ & $\begin{array}{l}\text { Pilot } \\
\text { Follow-up }\end{array}$ & $\begin{array}{l}3.910^{\mathrm{a}} \\
-.993^{\mathrm{a}}\end{array}$ & $\begin{array}{l}-.953 \\
-5.342\end{array}$ & $\begin{array}{l}8.772 \\
3.356\end{array}$ \\
\hline AH: kept monitor with daily habit $(\mathrm{N}=14)$ & $\begin{array}{l}\text { Pilot } \\
\text { Follow-up }\end{array}$ & $\begin{array}{l}16.713^{\mathrm{a}} \\
7.816^{\mathrm{a}}\end{array}$ & $\begin{array}{l}9.747 \\
1.586\end{array}$ & $\begin{array}{l}23.679 \\
14.047\end{array}$ \\
\hline
\end{tabular}

a. Covariates appearing in the model are evaluated at the following values: baseline Consumption $=3614.52 \mathrm{kWh}$.

\section{Table 4. Estimated Marginal Means (\% energy savings)}

There was a marginally significant effect over time (Wilks Lambda $=.94, F(1,50)=3.30$, $p=0.075$, partial eta squared $=.06$ ) with the savings of all groups decreasing over time. All groups sustained savings during the initial trial (table 4). This however appears to be due to subgroup specific characteristics as there is a significant between-subjects effect $(F(2,50)$ $=4.63 p=.014)$. As visible in table 5 , the difference in savings for households who had kept the monitor and developed a daily habit $(\mathrm{AH})$ was already significantly different during the initial trial in comparison to households who handed the monitor back (B) $(p=.004)$ but also in comparison to households who kept the monitor but didn't develop a habit $(\mathrm{ANoH})(p=.050)$. After the follow-up the difference is only significant between $\mathrm{B}$ and $\mathrm{AH}(p=.024)$. The group that formed a habit still had significant savings after the follow-up. Even though this cannot be conclusively attributed to the interventions with the energy monitor, it is highly likely that these contributed to the high savings of the receptive households who formed a daily habit, however the effects are still relatively short term as the same rate of fallback still occurs. 


\begin{tabular}{|c|c|c|c|c|c|c|}
\hline \multirow{2}{*}{80} & \multirow[b]{2}{*}{ Parameter } & \multirow[b]{2}{*}{ B } & \multirow[b]{2}{*}{$T$} & \multirow[b]{2}{*}{ Sig. } & \multicolumn{2}{|c|}{$95 \%$ Confidence Interval } \\
\hline & & & & & Lower Bound & Upper Bound \\
\hline Savings pilot & $\begin{array}{l}\text { Intercept } \\
\text { baseline consumption } \\
\text { ANoH: kept monitor w/o habit } \\
\text { B: handed monitor back in } \\
\text { AH: kept monitor with habit }\end{array}$ & $\begin{array}{l}22.061 \\
-.001 \\
-10.393 \\
-12.804 \\
0^{\mathrm{a}}\end{array}$ & $\begin{array}{l}3.963 \\
-1.081 \\
-2.012 \\
-3.025\end{array}$ & $\begin{array}{l}.000 \\
.285 \\
.050 \\
.004\end{array}$ & $\begin{array}{l}10.880 \\
-.004 \\
-20.769 \\
-21.306\end{array}$ & $\begin{array}{l}33.241 \\
.001 \\
-.018 \\
-4.301\end{array}$ \\
\hline Savings follow & $\begin{array}{l}\text { Intercept } \\
\text { Pre-pilot consumption } \\
\text { ANoH: kept monitor w/o habit } \\
\text { B: handed monitor back in } \\
\text { AH: kept monitor with habit }\end{array}$ & $\begin{array}{l}10.848 \\
.000 \\
-6.077 \\
-8.810 \\
0^{\mathrm{a}}\end{array}$ & $\begin{array}{l}2.179 \\
-686 \\
-1.315 \\
-2.327\end{array}$ & $\begin{array}{l}.034 \\
.496 \\
.194 \\
.024\end{array}$ & $\begin{array}{l}.848 \\
-.003 \\
-15.357 \\
-16.415\end{array}$ & $\begin{array}{l}20.848 \\
.002 \\
3.203 \\
-1.204\end{array}$ \\
\hline
\end{tabular}

a. This parameter is set to zero because it is redundant.

Table 5. Parameter estimates (in \% energy savings)

\section{Conclusions}

The first hypothesis; "At the end of the initial four-month trial, participants have an overall mean electricity saving," is confirmed. During the initial trial, the average savings that were achieved for all participants was $7.8 \%(S D=13.8)$. If the study had ended there, as most studies do, it would have been a sizable outcome for the interventions. As stated in the introduction, certain energy monitors advertise with similarly achieved figures.

A subgroup of highly motivated people (the same people that later reported development of habitual behaviour with the monitor) even managed to reach an initial electricity saving of $16.7 \%$, in comparison with $6.3 \%$ and $3.9 \%$ of the other groups (see table 4 ).

The second hypothesis was: "Participants' initial (four-month) electricity savings is not 
expected to be sustained over the entire 15-month period. However, participants who kept the monitor at home after the four-month initial trial are expected to better sustain their electricity savings than participants who returned the monitor."

This hypothesis is falsified. None of the groups sustained their savings, and the fallback rates in electricity savings of the three groups $(\mathrm{AH}, \mathrm{ANoH}$ and $\mathrm{B})$ do not differ significantly from each other. Participants who kept the monitor, though having liked it enough to retain it, did not manage to sustain their electricity savings any better than the people without a monitor.

Finally, the third hypothesis: "There is a positive relationship between having a daily habit of checking the monitor and sustaining the electricity savings over the 15-month period," could not be confirmed. Although the group that developed a habit $(\mathrm{AH})$ still had significant savings after the 15-month period (compared to group B), this cannot be attributed to habitual behaviour, as this group $(\mathrm{AH})$ already showed more than average savings during the initial four-month trial. A more likely interpretation is that the savings were due to subgroup specific characteristics (i.e. personality traits): these participants were probably predisposed and more receptive to the interventions with the energy monitor than the other participants.

It should be noted that other variables outside this study could have contributed to the participants' electricity savings, such as government campaigns, or the economic crisis. It is however highly likely that the energy monitor was an effective trigger for those who saved electricity. Although it is hard to generalize these findings to other household energy monitors, based on the results of this case study, we would like to draw the following tentative conclusions:

- Interventions that focus on energy monitors are mainly successful if they are targeted at a specific niche of users who are highly motivated to incorporate the use of the monitor into their daily lives. The exact personality traits of these users are yet to be determined. 
- An energy monitor is not effective over a longer period ( $>4$ months) for a majority of users. The participants involved in the case study could not sustain the initial reductions in electricity consumption over a period of 15 months.

Possible explanations for the lack of sustained savings may be one or a combination of the following:

- People fall back in old behaviour patterns.

- Over time, people add new appliances to their household. There is some evidence of this in the online surveys (reports of buying plasma screens, air-conditioning and electric heaters). Even if people have developed a habit of electricity saving behaviour, these new products may lead to an overall increase in electricity consumption, as an energy monitor mainly curtails existing behaviour.

- The so-called rebound effect. People who substitute inefficient products for more efficient ones sometimes increase the use of these more efficient products (Terpstra, 2008), thus negating the savings.

\section{Discussion}

Certain shortcomings in the design of the research could not be prevented within this study. The case study was conducted in joint cooperation with commercial parties, which has benefits for directly implementing research into practice, but also posed some scientific limitations. It could for instance not be ascertained, beyond probable cause, that the chosen intervention method (energy monitor) was completely responsible for the savings. As in many other studies, this case study also had to deal with convenience sampling. Participants' awareness that their electricity consumption was being monitored during the initial trial might have contributed to the higher savings (Hawthorne effect), although four months is a long time to consciously register this. In contrast, participants did not know that their electricity consumption would be monitored in the period after the initial trial (the follow-up). 
Furthermore, relying on questionnaires and self reported meter data introduces a high error rate in the raw data. Tracking the real-time usage of energy monitors in future studies would give more reliable insights.

\section{Overall conclusions}

Both the literature review and the case study highlighted a number of focal points, which have implications for future research. The case study confirms the need for longitudinal research, as it shows how the initial effectiveness of feedback tends to wear off over a longer period of time ( $>4$ months). A second finding is that certain groups of people seem more receptive to energy saving interventions than others. Obviously, a 'one-size-fits-all' approach for home energy monitors cannot be justified. In the development of energy monitors, their design should take into account that user's responses to a type of intervention are divergent. However, the effectiveness of energy monitors could potentially be enhanced by using insights from interaction design research, such as persuasive technology (as discussed in the literature review) or by considering the night-time ritual of checking the monitor (as discussed in the case study) as part of the design strategy. The differences in savings between households are of such a magnitude that interventions tailored to individual households are also recommended.

Likewise, the differences in achieved -and sustained- savings between users indicates that a fuller understanding of the influence of contextual factors, design, usability, and users' characteristics and/or ecological attitudes is highly desirable. Within social science attention for participant's characteristics and attitudes is evident, but other factors receive too little attention. As Abrahamse et al.(2005) also concluded, demographics have repeatedly served as classification but other attributes are less well documented. Exchange of knowledge between designers, Hems practitioners, and social scientists when instigating studies and campaigns therefore needs to be encouraged. 
Further research should also consider the development and testing of Hems that manage energy (rather than 'just' monitor it), as was introduced in the section on background relations. These allow users control when appliances and installations consume energy, while subtly correcting their undesired behaviours. This leads to the question: To what extent does more active control by users result in more persistent savings?

Finally, the case study didn't allow for the testing of variations in the design of the energy monitor. However, this is definitely an avenue worth further exploration.

While several leads have been introduced both in the literature review as well as in the case study, the focus should be on testing and finding the right balance in types of intervention and Hems's design strategy, coupled with a clear idea of the kind of user the monitor is serving.

\section{Acknowledgements}

The authors are grateful for the valuable comments of the four anonymous Building Research \& Information referees and the Editors. Many thanks also to the companies who participated in the trial.

\section{Bibliography}

Abrahamse, W., Steg, L., Vlek, C. \& Rothengatter, T. (2005) A review of intervention studies aimed at household energy conservation. Journal of Environmental Psychology 25, 273-291.

Borgmann, A. (1995) The Depth of Design. IN BUCHANAN, R. \& MARGOLIN, V. (Eds.) Discovering Design: Explorations in Design Studies. Chicago, University of Chicago Press, 13-22.

Brohus, H., Heiselberg, P., Simonsen, A. \& Sørensen, K. C. (2009) Uncertainty of energy consumption assessment of domestic buildings. Proceedings of the Eleventh International IBPSA Conference. Glasgow, Schotland.

Cialdini, R. B. (1993) Influence: science and practice, New York, HarperCollins.

Crosbie, T. \& Baker, K. (2010) Energy-efficiency interventions in housing: learning from the inhabitants. Building Research \& Information, 38(1), 70 - 79.

Darby, S. (2006) The effectiveness of feedback on energy consumption. A review for DEFRA of the literature on metering, billing and direct displays. Environmental Change Institute, University of Oxford.

Delft University of Technology (2009) a Mission Statement [online] http://www.tudelft.nl/live/pagina.jsp?id=b4c76e5e-3a59-4be9-a050c847d3a5fbb2\&lang=en [accessed september 2009]

Firet L.T. (2009) Interview concerning PowerPlay pilot Nuon, SenterNovem and UCPartners [Interview] (personal communication, 29-06-2009).

Fischer, C. (2008) Feedback on household electricity consumption: a tool for saving energy? Energy Efficiency, 1(1), 79-104. 
Fogg, B. J. (2003) Persuasive Technology: Using Computers to Change What We Think and Do, Morgan Kaufmann.

Fogg, B. J. (2009) A Behavior Model for Persuasive Design. Persuasive 2009, The 4th International Conference on Persuasive Technology. Claremont, California, USA.

Goldstein, N. J., Cialdini, R. B. \& Griskevicius, V. (2008) A room with a viewpoint: Using social norms to motivate environmental conservation in hotels. Journal of Consumer Research, 35, 472-482.

Ihde, D. (1990) Technology and the Lifeworld From Garden to Earth Indiana, Indiana University Press.

International Energy Agency (lea) (2009) Gadgets and Gigawatts, Policies for Energy Efficient Electronics. Paris, International Energy Agency

Kidd, A. \& Williams, P. (2008) The Talybont Trial, exploring the psychology of smart meters. Brecon, Wales, The Prospectory.

Liikkanen, L. (2009) Extreme-user approach and the design of energy feedback systems. International Conference on Energy Efficiency in Domestic Appliances and Lighting 2009 (EEDAL'09),. Berlin, Germany.

Lockton, D. (2009) The Design with Intent Toolkit v.0.9 [online] http://architectures.danlockton.co.uk/2009/04/06/the-design-with-intent-toolkit/ [accessed 01-09-2009]

Lockton, D., Harrison, D. \& Stanton, N. (2008) Design with Intent: Persuasive Technology in a Wider Context. Persuasive Technology. 274-278.

Lofstrom, E. \& Palm, J. (2008) Visualising household energy use in the interest of developing sustainable energy systems. Housing Studies, 23(6), 935-940.

Matsukawa, I. (2004) The Effects of Information on Residential Demand for Electricity. Energy Journal, 25(1), 1-17.

Mccalley, L. T. \& Midden, C. J. H. (2002) Energy conservation through product-integrated feedback: The roles of goal-setting and social orientation. Journal of Economic Psychology, 23(5), 589-603.

Midden, C. \& Ham, J. (2009) Using negative and positive social feedback from a robotic agent to save energy. Proceedings of the 4th International Conference on Persuasive Technology. Claremont, California, ACM.

Milieu Centraal (2005) Persbericht 29 October: Mensen gebruiken minder stroom door energiemeter (Press release 29 October: People use less electricity because of energy meter) [online] www.milieucentraal.nl [accessed dec 2008]

Milieu Centraal (2008) Gas en Elektriciteitverbruik gemiddeld huishouden (average gas and electricity consumption Dutch household) [online] www.milieucentraal.nl [accessed March 2008]

Mountain, D. (2006) The Impact of Real-Time Feedback on Residential Electricity Consumption: The Hydro One Pilot. Ontario, Mountain Economic Consulting and Associates, Inc.

Terpstra, P. M. J. (2008) Consument-en-technologie in het perspectief van duurzame ontwikkeling, Afscheidsrede uitgesproken op 15 mei 2008, Wageningen, Wageningen Universiteit.

Ueno, T., Inada, R., Saeki, O. \& Tsuji, K. (2006a) Effectiveness of an energy-consumption information system for residential buildings. Applied Energy, 83(8), 868-883.

Ueno, T., Sano, F., Saeki, O. \& Tsuji, K. (2006b) Effectiveness of an energy-consumption information system on energy savings in residential houses based on monitored data. Applied Energy, 83(2), 166-183.

Van Houwelingen, J. H. \& Van Raaij, W. F. (1989) The Effect of Goal-Setting and Daily Electronic Feedback on In-Home Energy Use The Journal of Consumer Research 16(1), 98-105.

Wood, G. \& Newborough, M. (2006) Energy-use information transfer for intelligent homes: Enabling energy conservation with central and local displays. Energy and Buildings, 39(4), 495-503. 